\title{
EXACT TOPOLOGICAL DENSITY IN THE LATTICE SKYRME MODEL*
}

\author{
BENJAMIN SVETITSKY \\ School of Physics and Astronomy, Raymond and Beverly Sackler Faculty of Exact \\ Sciences, Tel Aviv University, 69978 Tel Aviv, Israel \\ E-mail: bqs@julian.tau.ac.il \\ ALEC J. SCHRAMM \\ Department of Physics, Occidental College, Los Angeles, California 90041, USA \\ E-mail: alec@oxy.edu
}

\begin{abstract}
We propose using the Skyrme model on a lattice as an effective field theory of meson-baryon interactions. To this end we construct a local topological density that involves the volumes of tetrahedra in the target space $S^{3}$ and we make use of Coxeter's formula for the Schläfli function to implement it. We calculate the mean-square radius of a skyrmion in the three-dimensional Skyrme model, and find some surprises.
\end{abstract}

\section{Why a lattice Skyrme model?}

The Skyrme model is a theory of a scalar field $U(x) \in S U(2)$ with the action

$$
S=\int d \mathbf{x}\left[\frac{f_{\pi}^{2}}{16} \operatorname{Tr}\left|\partial_{\mu} U\right|^{2}-\frac{1}{32 e^{2}} \operatorname{Tr}\left(\left[\partial_{\mu} U U^{\dagger}, \partial_{\nu} U U^{\dagger}\right]^{2}\right)\right] .
$$

The action possesses an $S U(2) \times S U(2)$ chiral symmetry which is spontaneously broken. The Goldstone bosons are taken to represent pions, massless unless we add a symmetry-breaking term to $S$. The theory also contains solitons, stabilized by the 4-derivative Skyrme term shown, that have the properties of baryons. The model furnishes a rich phenomenology of pion-nucleon interactions at low momenta.

The Skyrme model does not really exist as a continuum theory, since the action is non-renormalizable. For this reason, existing treatments of the model are semiclassical, quantizing only the collective degrees of freedom of the soliton. Full quantization of the theory requires a cutoff. In considering the sector without skyrmions, this cutoff can be removed order by order in perturbation theory at the price of an ever-lengthening list of higher-dimension counterterms. In the skyrmion sector, however, even this is difficult to accomplish.

* Presented at the International Workshop on Strong and Electroweak Matter (SEWM 2000), Marseille, France, 14-17 June 2000. 
We suggest 1 that the limitations of the "continuum" model be turned to advantage by treating the Skyrme model as an effective field theory without removing the cutoff. Now the cutoff is part of the specification of the theory. The form of $S$ may be chosen freely, subject only to phenomenological tests.

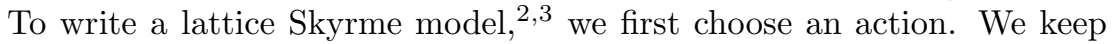
the cutoff-the lattice spacing - fixed. We apply the full panoply of lattice methods to calculate quantities of interest, going beyond semiclassical methods and beyond perturbation theory. Renormalization consists of adjusting the bare couplings and the lattice spacing to match physical parameters. No continuum limit is necessary - or possible.

\section{Lattice topology}

Continuum solitons are classified by the winding number of the field configuration that maps the compactified 3-space $\left\{R^{3}+\infty\right\}$ to the field space $S U(2)$, both of which are topologically equivalent to the 3 -sphere $S^{3}$. The differential volume in the target space is given by

$$
d \tau=\frac{1}{24 \pi^{2}} \epsilon_{i j k} \operatorname{Tr}\left(U^{-1} \partial_{i} U\right)\left(U^{-1} \partial_{j} U\right)\left(U^{-1} \partial_{k} U\right) d^{3} r
$$

which integrates to an integer $n$. On a lattice, we calculate this differential volume directly as follows.

1. We cut each lattice cube into five tetrahedra:

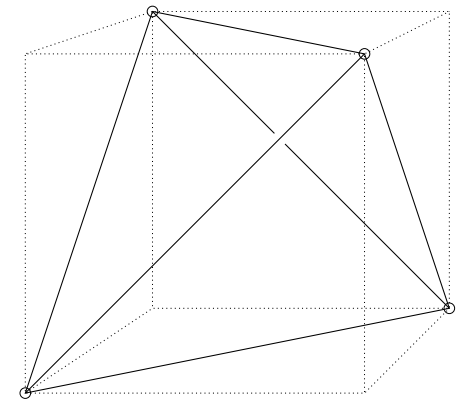

2. Each tetrahedron maps to a tetrahedron in $S^{3}$. Oddly enough, there is no simple formula for the volume of this curved tetrahedron. 9 Our solution to this problem begins by dropping perpendiculars to cut the

${ }^{a}$ Cf. Girard's theorem for a triangle in $S^{2}$, which says that the area is equal to the angular excess. 
curved tetrahedron into six quadrirectangular tetrahedra (qrt's), of which we show two:

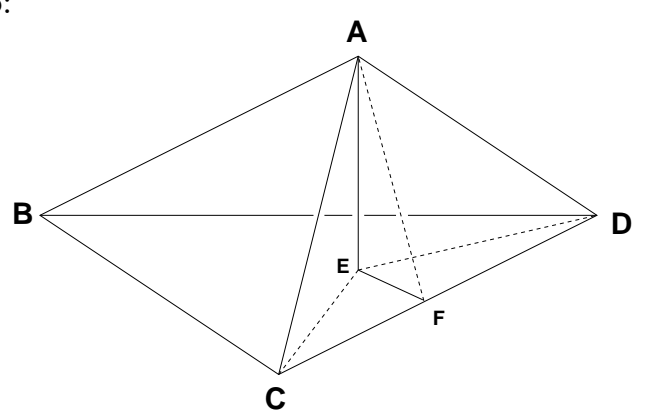

In general, a qrt can be constructed by drawing three mutually perpendicular line segments $\mathbf{P}_{0} \mathbf{P}_{1} \mathbf{P}_{2} \mathbf{P}_{3}$ and connecting all the vertices:

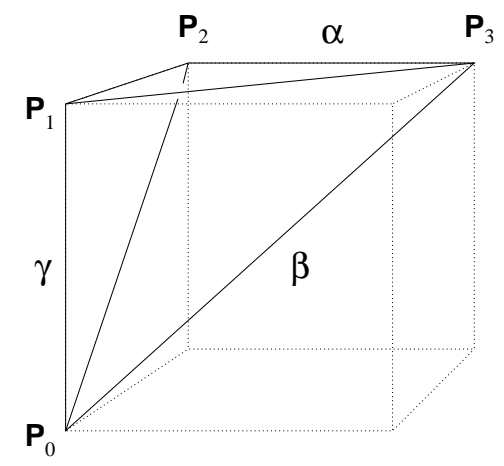

(This works in $S^{3}$ as well as well as in $R^{3}$.) All the faces of a qrt are right triangles. Three of the dihedral angles of a qrt are right angles; the other three may be labeled $\alpha, \beta, \gamma$.

3. Polytopes in $S^{n}$ were studied by Schläfli in the 19th century, and in 1858 he wrote down a differential equation for the volume of a qrt as a function of $\alpha, \beta, \gamma$. This equation was solved by Coxeter in 1935 . The volume $V$ is given by the Schläfli function $S$ according to

$$
\begin{aligned}
V(\alpha, \beta, \gamma)= & \frac{1}{4} S\left(\frac{\pi}{2}-\alpha, \beta, \frac{\pi}{2}-\gamma\right) \\
S(x, y, z) \equiv \sum_{m=1}^{\infty} & \left(\frac{D-\sin x \sin z}{D+\sin x \sin z}\right)^{m} \frac{\cos 2 m x-\cos 2 m y+\cos 2 m z-1}{m^{2}} \\
& -x^{2}+y^{2}-z^{2}
\end{aligned}
$$


where $D=\sqrt{\sin ^{2} \alpha \sin ^{2} \gamma-\cos ^{2} \beta}$ is the "angular excess" - which vanishes for a Euclidean qrt. In fact $V \rightarrow 0$ as $D \rightarrow 0$, as expected.

\section{The lattice action}

The topological density defined above will not be meaningful until we resolve ambiguities associated with what is known as "topology dropping through the lattice." A field configuration $U(x)$ specifies the vertices of all the tetrahedra in $S^{3}$. Given the vertices of a tetrahedron, however, there are two ways to define its interior: either that part of $S^{3}$ that includes the north pole (for example), or that part that doesn't. Taking one of these to be positive, say $0<V<1$ (in units of $2 \pi^{2}$, the volume of $S^{3}$ ), the other will be $V-1<0$. Thus we cannot simply calculate the winding number of a given field configuration. We can resolve this ambiguity by defining the volume of a tetrahedron to satisfy always $|V|<1 / 2$.

But now consider a tetrahedron whose volume is $1 / 2-\epsilon$. A small change in one of the vertices can push its volume to $1 / 2+\delta$, which will now be interpreted as $-1 / 2+\delta$, while the volumes of its neighbors change by $\delta+\epsilon$. Thus the winding number by our convention has jumped by 1: Topology has fallen through the lattice. The only way to avoid this is to force the field configurations to be sufficiently smooth that a tetrahedron volume stays well away from $\pm 1 / 2$. We can do this by choosing a sufficiently stiff kinetic term, such as 4

$$
S_{1}=(\alpha-1) \sum_{\mathbf{n} \mu} \log \left(\mathbf{a}_{\mathbf{n}} \cdot \mathbf{a}_{\mathbf{n}+\hat{\mu}}-\alpha\right),
$$

with $U=a_{0}+i a_{i} \sigma_{i}$. This constrains $\mathbf{a} \cdot \mathbf{a}^{\prime}>\alpha$. We find that setting $\alpha=0.1$ keeps the tetrahedra well away from ambiguity.

With the action (5) alone, solitons will collapse to radii on the order of the lattice spacing before they notice that the action isn't quadratic in derivatives (à la Derrick's Theorem). To make sizable skyrmions possible, we add a Skyrme term

$$
\begin{aligned}
S_{2}=4 \sum_{\mathbf{n}} \sum_{\mu>\nu}\left\{\left(\mathbf{a}_{\mathbf{n}+\hat{\mu}}-\mathbf{a}_{\mathbf{n}+\hat{\nu}}\right)^{2}\left(\mathbf{a}_{\mathbf{n}+\hat{\mu}+\hat{\nu}}-\mathbf{a}_{\mathbf{n}}\right)^{2}\right. \\
\left.-\left[\left(\mathbf{a}_{\mathbf{n}+\hat{\mu}}-\mathbf{a}_{\mathbf{n}+\hat{\nu}}\right) \cdot\left(\mathbf{a}_{\mathbf{n}+\hat{\mu}+\hat{\nu}}-\mathbf{a}_{\mathbf{n}}\right)\right]^{2}\right\} .
\end{aligned}
$$

The total action is $S=\beta_{1} S_{1}+\beta_{2} S_{2}$.

Will the system still tunnel between topological sectors? In principle, yes. A non-local updating scheme could create a smooth skyrmion at a blow, 
which would change the winding number by 1 . This won't happen with a local algorithm, such as local Metropolis updating.

This determines how to choose an updating scheme. Thus, if we are interested in doing thermodynamics with a chemical potential, summing over topological sectors according to

$$
Z(\mu)=\sum_{n} e^{\mu n} Z_{n},
$$

we would choose a scheme that does nucleate smooth skyrmions (or even point-like defects). Our interest, however, is in studying the properties of a single skyrmion, so we trap it in our computer by insisting on local updating that preserves winding number.

\section{The Skyrme model in three dimensions, and the future}

As a first application, we have looked 10 at the three-dimensional Skyrme model, which may be thought of as a Ginzburg-Landau theory for the full quantum theory at nonzero temperature. The bare couplings in the action (at fixed cutoff) depend on physical parameters such as $f_{\pi}$, the baryon mass, and the temperature, but we have not yet tackled this matching problem. We ran Monte Carlo simulations in the single-skyrmion sector and measured the mean-square radius $\left\langle R^{2}\right\rangle$ of the topological density defined above. We found, to our surprise, that our lattice action $S$ admits a multitude of metastable minima, configurations of the skyrmion that have (generally) smaller radii than the skyrmion that actually minimizes the action. As the "temperature" of the $3 \mathrm{~d}$ model-namely, $1 / \beta_{1}$ for fixed $\beta_{2} / \beta_{1}$-is raised, more of these local minima come into play and the skyrmion actually ends up shrinking as it is heated. Recall that in this Ginzburg-Landau theory, this temperature reflects both thermal and quantum fluctuations of the $4 \mathrm{~d}$ quantum theory. It is possible that renormalization of the ratio $\beta_{2} / \beta_{1}$ will destroy this effect.

Directions for further study include simulation of the full $4 \mathrm{~d}$ theory and renormalization of its couplings (at fixed cutoff); study of the dependence of the size and shape of the equilibrium skyrmion on the physical temperature and density; and measuring the sensitivity of various results to the chosen form of the lattice action.

\section{Acknowledgments}

This work was supported by the Israel Science Foundation under Grant No. 255/96-1 and by the Research Corporation.

Skyrme2: submitted to World Scientific on November 21, 2018 


\section{References}

1. A. J. Schramm and B. Svetitsky, hep-lat/0008003.

2. R. Saly, Phys. Rev. D 31, 2652 (1985).

3. C. DeTar, Phys. Rev. D 42, 224 (1990).

4. R. S. Ward, Lett. Math. Phys. 35, 385 (1995) hep-th/9502048. 